\title{
Characteristics and management of immune- related adverse effects associated with ipilimumab, a new immunotherapy for metastatic melanoma
}

\author{
Stephanie Andrews' \\ Rita Holden ${ }^{2}$ \\ 'H Lee Moffitt Cancer Center \\ and Research Institute, Tampa, FL, \\ ${ }^{2}$ St Luke's Hospital and Health \\ Network Cancer Center, Easton, \\ PA, USA
}

Video abstract

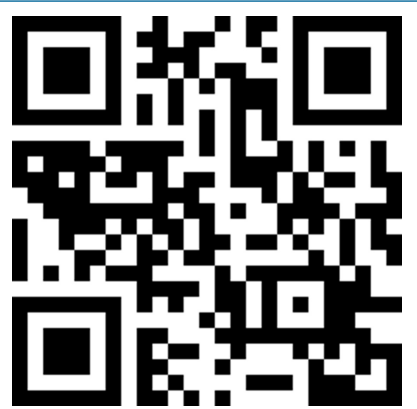

Point your SmartPhone at the code above. If you have a QR code reader the video abstract will appear. Or use: http://dvpr.es/ONHuTB

This article was published in the following Dove Press journal:

Cancer Management and Research

II September 2012

Number of times this article has been viewed

Abstract: When diagnosed in its early stages, melanoma is highly treatable and associated with good long-term outcomes; however, the prognosis is much poorer for patients diagnosed with advanced or metastatic melanoma. For decades, available treatments were effective in only a few patients and associated with significant safety concerns. Ipilimumab is a novel immunotherapy which has proved to be an exciting breakthrough in the treatment of melanoma. It is the first drug approved for the treatment of melanoma by the Food and Drug Administration (FDA) which has shown a survival benefit in a randomized Phase III clinical trial. The objective of this review is to provide information on the administration, treatment responses, and expected outcomes of treatment of metastatic melanoma with the new immunotherapeutic agent, ipilimumab, a drug with a unique mechanism of action that differentiates it from current treatments. Guidelines for the management of immune-related adverse events associated with ipilimumab therapy are also presented. These stress vigilance, prompt intervention, and the use of corticosteroids as appropriate. Various ipilimumab-associated immune-related adverse events, both common (enterocolitis, dermatitis) and less frequent (hepatitis, hypophysitis), are illustrated in case studies. Nurses are uniquely positioned to provide patient and caregiver education on how this new therapy differs from traditional cytotoxic agents, to recognize the signs and symptoms of immune-related adverse events, and to report them immediately, and finally, to be aware of the patterns of response that are commonly observed in patients receiving ipilimumab therapy.

Keywords: melanoma, ipilimumab, immunotherapy, case studies

\section{Introduction}

\section{Prevalence and incidence of melanoma}

Melanoma of the skin is the sixth most common cancer type reported in US cancer registries. ${ }^{1}$ Of the estimated 70,230 new cancer cases (38,870 men and 29,260 women), 8790 deaths were attributed to melanoma in the US in $2011 .^{2}$ Approximately $84 \%$ of cases present with local disease (stages I and II), $8 \%$ of patients have regional disease, and only $4 \%$ show distant metastases, with $4 \%$ being unstaged. ${ }^{3}$

\section{Mortality in advanced melanoma}

Melanoma staging is based on many factors, including Breslow thickness, ulceration, mitotic rate, extent of nodal involvement, distant disease site, and in-transit disease. Advanced melanoma or unresectable stage III disease includes extensive nodal involvement but is limited to a single basin. Stage IV disease is defined as distant skin involvement, soft tissue involvement, lung, and/or visceral sites. ${ }^{4}$ Survival rates are highly dependent on the stage of metastatic disease, ${ }^{3}$ with stage IV melanoma patients showing an approximately $15 \%$ survival rate at 5 years. ${ }^{4,5}$ 
Metastasis to the central nervous system occurs in over $50 \%$ of patients with advanced melanoma. Furthermore, mortality from melanoma metastasizing to the central nervous system or brain is particularly high, with a median overall survival of 4.4 months and a 5 -year survival rate of $3 \%{ }^{6-10}$

\section{Therapeutic options for melanoma}

Surgical options are limited to patients with a small number of lesions who can be rendered free of disease or can be used for palliation. Unfortunately, traditional chemotherapy and multiple surgeries demonstrate marginal utility. In addition to surgical resection, radiotherapy may be considered for palliation or symptom management. ${ }^{5}$

Current clinical treatment guidelines for systemic therapy of unresectable or metastatic melanoma underscore the need for definitive recommendations concerning effective therapy in light of the tenacious nature of the disease and relative ineffectiveness of existing therapeutic options. . $^{511}$ Other than entry into a clinical trial, treatment with dacarbazine or high-dose interleukin-2 was the only therapy approved by the US Food and Drug Administration (FDA) until recently. Combination sequential biochemotherapy regimens (eg, dacarbazine, cisplatin, vinblastine + interleukin-2, and interferon alpha2b) have not shown a significant overall survival benefit compared with chemotherapy alone and are also associated with greater toxicity. ${ }^{12,13}$

Vemurafenib (Zelboraf ${ }^{\circledR}$, Genentech, South San Francisco, CA) is a BRAF inhibitor that has demonstrated activity in patients with metastatic melanoma who harbor the V600E BRAF mutation. Recent interim results of a Phase III study have reported 6-month overall survival of $84 \%$ (95\% confidence interval 78-89) in patients receiving vemurafenib compared with $65 \%$ in the dacarbazine arm ( $95 \%$ confidence interval 56-73) of the study. ${ }^{14}$ The rate of progression-free survival was also improved in the vemurafenib arm, and this agent has received approval from the FDA for the treatment of patients with unresectable or metastatic melanoma whose tumors harbor the V600E mutation. ${ }^{14,15}$ However, the long-term efficacy and safety of vemurafenib has yet to be determined.

Ipilimumab (Yervoy ${ }^{\mathrm{TM}}$, Bristol-Myers Squibb, Princeton, $\mathrm{NJ}$ ) at a dose of $3 \mathrm{mg} / \mathrm{kg}$ was approved for treatment of unresectable or metastatic melanoma by the FDA in March of 2011 based primarily on the findings of the MDX010-20 registration study. ${ }^{16}$ Ipilimumab is an immunotherapy drug which has proven to be an exciting breakthrough in the treatment of melanoma. It is the first drug approved for the treatment of melanoma by the FDA which has shown a survival benefit in a randomized Phase III study. Ipilimumab was also approved by the European Medicines Agency and the Therapeutic Good Association in Australia for treatment of unresectable stage III or stage IV melanoma in previously treated patients.

Ipilimumab is a monoclonal antibody that blocks cytotoxic $\mathrm{T}$ lymphocyte antigen- 4 , an inhibitor of $\mathrm{T}$ cell activation, thereby potentiating an immune response. ${ }^{17}$ Its unique mechanism of action differentiates it from chemotherapies, in that it targets the immune system rather than directly targeting the tumor itself. Clinical overall survival benefit was demonstrated with ipilimumab when compared with a vaccine against glycoprotein 100 in patients with unresectable or metastatic melanoma in a pivotal Phase III study (MDX010-20). Patients receiving ipilimumab survived 4 months longer on average than those receiving the vaccine. ${ }^{16}$ One-year and two-year survival rates for ipilimumab $3 \mathrm{mg} / \mathrm{kg}$ monotherapy were $45 \%$ and $23 \%$, respectively, which is nearly double the survival rate of the control arm of the study. ${ }^{16}$

A second Phase III study of ipilimumab in treatmentnaïve patients with unresectable stage III or IV melanoma was recently published. ${ }^{18}$ This study was done using a higher, experimental dose of ipilimumab at $10 \mathrm{mg} / \mathrm{kg}$ in combination with dacarbazine compared with dacarbazine alone. The combination arm of the study showed significantly higher survival rates in patients who received dacarbazine alone at one year ( $47.3 \%$ versus $36.3 \%)$, 2 years $(28.5 \%$ versus $17.9 \%)$, and 3 years $(20.8 \%$ versus $12.2 \%$, hazard ratio for death $0.72 ; P<0.001)$. The study not only confirmed the overall survival benefit of ipilimumab but also demonstrated a similar safety profile, although the rates of specific safety events varied from the MDX010-20 study. ${ }^{16,18}$

\section{Summary of pharmaceutical treatment options}

- Dacarbazine has low toxicity, is well tolerated, minimally effective, and has limited progression-free survival

- High-dose interleukin-2 has high toxicity, is used in highly selected patients, and has limited efficacy

- Combination biochemotherapeutic regimens have not been shown to have an overall survival benefit, and have added toxicity

- Vemurafenib shows a rapid response, has improved 6-month overall survival and progression-free survival, but lacks durability

- Ipilimumab has a unique side effect profile, and has been shown to improve one-year and two-year overall survival. 


\section{Ipilimumab treatment regimen}

Ipilimumab is administered as a 90 -minute intravenous infusion at a dose of $3 \mathrm{mg} / \mathrm{kg}$ every 3 weeks for four doses over an induction period of 12 weeks. ${ }^{19}$ In the registration trial, some patients who met certain criteria (stable disease for 3 months' duration after week 12 or a partial or complete response) were offered reinduction therapy. Scans for tumor assessment are repeated 2 weeks after the fourth treatment dose (week 12) and in 12 weeks (week 24) and every 3 months thereafter. If the patient has a mixed response or questionable progression of disease at any point then clinical judgment should be exercised and a repeat scan in 4-6 weeks is prudent.

\section{Immune-related AEs with ipilimumab}

The most common safety events associated with ipilimumab therapy are immune-related; a recent pooled analysis of 14 completed Phase I-III ipilimumab clinical trials showed that $64.2 \%$ of patients experienced an immune-related adverse event (AE) of any grade. ${ }^{20}$ Immune-related AEs are likely reflective of the immune-based mechanism of action of ipilimumab and may affect various organs. An overview of the rate of immune-related AEs associated with ipilimumab $3 \mathrm{mg} / \mathrm{kg}$ in the Phase III MDX010-20 registration trial is shown in Table 1A. ${ }^{16}$ The most common immune-related AEs included toxicities of the skin, gastrointestinal tract, endocrine system, and liver. ${ }^{16}$

The time to onset of immune-related AEs associated with ipilimumab in the Phase III MDX010-20 study and a pooled analysis of patients from the Phase II studies (CA184-004, CA184-007, CA184-008, and CA184-022) are shown in Table 1B. ${ }^{11,16}$ These analyses demonstrate that the majority of immune-related AEs initially manifest during induction phase; however, a minority occurs weeks to months after discontinuation of ipilimumab. ${ }^{19}$ Time to resolution of immune-related AEs experienced by patients varied from 4.3 to 7.7 weeks on average across all studies. However, time to recovery may be expedited when patients and caregivers report early signs of adverse events promptly. ${ }^{11,16}$

\section{Skin immune-related AEs Case study I: immune-related dermatitis}

A 40-year-old woman with a melanoma on her back, Clark's level III, Breslow depth of $0.4 \mathrm{~mm}$, without ulceration, and with a modest lymphocytic infiltrate underwent wide local excision. Two years later, she developed a palpable lymphatic recurrence in her left axilla. She had a left axillary node dissection, with nine of 22 nodes being positive. Her tumor was HLA-A*0201-positive and staging studies were negative. She proceeded into an adjuvant clinical trial and was treated with peptide vaccine plus ipilimumab $3 \mathrm{mg} / \mathrm{kg}$, receiving three doses of vaccine and one dose of ipilimumab. Approximately 4 weeks following treatment with ipilimumab, she developed severe itching, with rash over more than $75 \%$ of her body, and pain and swelling in the entire upper leg at the site of her last injection as well as swelling of the lips. She was in severe pain and unable to move or sleep for two nights despite taking Benadryl ${ }^{\circledR}$ and topical steroid application prior to a visit to the emergency room. At the hospital, her husband took a photograph and sent it to her treating physician (Figure 1). She received intravenous methylprednisolone sodium succinate, and was treated with $60 \mathrm{mg}$ prednisone and recovery occurred within 4-5 days. In addition, the patient was given a slow 30-day taper of oral steroid. Because the patient presented with a dose-limiting grade 3 toxicity, treatment with ipilimumab was withheld. She has been followed with close observation and, to date, has no evidence of disease.

Table I A Rates of immune-related adverse events from the MDX0 I0-20 registration trial, which included previously treated patients with unresectable stage III or IV melanoma treated with ipilimumab $3 \mathrm{mg} / \mathrm{kg}$ alone, a control vaccine alone (glycoprotein I00), or a combination of both ipilimumab and glycoprotein 100

\begin{tabular}{|c|c|c|c|}
\hline $\begin{array}{l}\text { Immune-related AEs } \\
\text { (all grades) }\end{array}$ & $\begin{array}{l}\text { Ipilimumab + } \\
\text { glycoprotein } 100 \\
(n=380)\end{array}$ & $\begin{array}{l}\text { Ipilimumab alone } \\
(n=|3|)\end{array}$ & $\begin{array}{l}\text { Glycoprotein } 100 \text { alone } \\
(n=132)\end{array}$ \\
\hline Any, n (\%) & $380(58.2)$ & $|3|(6||)$. & | 32 (3|.8) \\
\hline Dermatologic, n (\%) & $380(40.0)$ & $|3|(43.5)$ & $132(16.7)$ \\
\hline Gastrointestinal, n (\%) & $380(31.1)$ & $|3|(29.0)$ & $132(14.4)$ \\
\hline Endocrine, n (\%) & $380(3.9)$ & $|3|(7.6)$ & $132(1.5)$ \\
\hline Hepatic, n (\%) & $380(2.1)$ & $|3|(3.8)$ & $132(4.5)$ \\
\hline
\end{tabular}

Notes: There was no statistically significant difference between the immune-related AEs in the two ipilimumab-containing arms; some patients had more than one immunerelated $\mathrm{AE}$.

Abbreviation: $\mathrm{AE}$, adverse event. 
Table IB Time to onset of immune-related adverse events in the Phase III MDX0I0-20 study, pooled Phase II studies at 3 mg/kg, and pooled Phase II studies at $10 \mathrm{mg} / \mathrm{kg}$

\begin{tabular}{|c|c|c|c|}
\hline & Phase III (3 mg/kg) & Phase II (3 mg/kg) & Phase II (10 mg/kg) \\
\hline irAEs grade $2-5, n$ & 42 & 38 & 142 \\
\hline Median weeks to onset of grade $2-5 \operatorname{irAE}(95 \% \mathrm{Cl})$ & $6.07(3.43-7.29)$ & $6.93(4.86-7.57)$ & $4.93(4.00-5.57)$ \\
\hline irAEs grade $2-4$ resolved, $n$ (\%) & $31(75.6)$ & $24(63.2)$ & $117(83.6)$ \\
\hline Median weeks to resolution of grade $2-4$ irAE, $n(95 \% \mathrm{Cl})$ & $6.29(4.29-8.43)$ & $5.7 \mid(2.14-15.1)$ & $5.07(3.86-7.14)$ \\
\hline
\end{tabular}

Note: Rate and time to resolution of grade 2-4 immune-related adverse events (grade 5 events are fatal) are also presented.

Abbreviation: irAE, immune-mediated adverse event.

\section{Management}

Dermatologic symptoms occurred in $40 \%$ of patients treated with ipilimumab in the MDX010-20 trial, ${ }^{16}$ with the majority of these immune-related AEs being classified as grades $1-2$. The most common grade 1 or 2 skin immune-related AEs with ipilimumab were dermatitis, with rash $(21.1 \%$ of patients) and pruritus (24.7\% of patients). Rash may be local or diffuse erythroderma of maculopapular origin, with or without pruritus. Patients should be advised to report any skin-related changes and monitor for rash and pruritus. Lubricants such as Aveeno ${ }^{\circledR}$ oatmeal lotion or Lubriderm ${ }^{\circledR}$ moisturizing cream may be used for grade 1-2 rash; treatment should be interrupted for grade 3 dermatitis until the rash returns to grade $1-2$; and grade 4 toxicity requires hospitalization. For mild or moderate dermatitis, topical and/or systemic corticosteroids may be used. Grade 3 to 4 skin immune-related AEs include symptoms of severe and fatal inflammation of the skin, including StevensJohnson syndrome and toxic epidermal necrolysis, ${ }^{19}$ which have been reported in rare instances of patients receiving ipilimumab. Ipilimumab should be withheld in patients with grades 3-4 toxicity and should be permanently discontinued

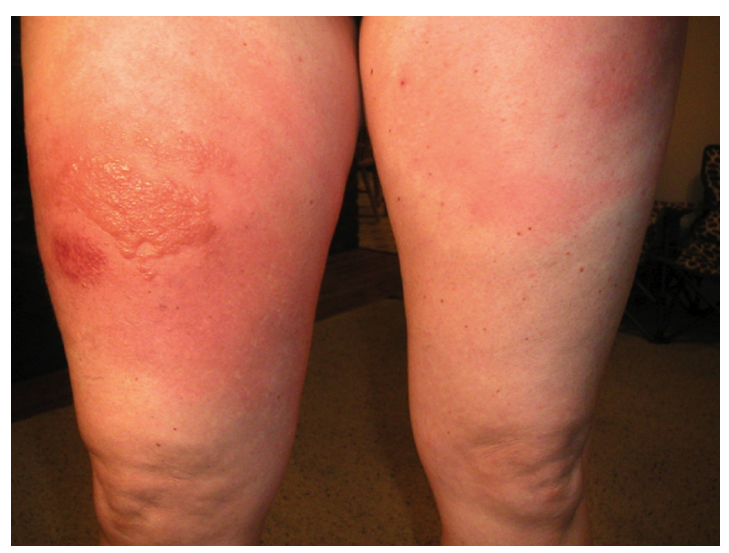

Figure I Patient on ipilimumab who experienced itching and rash over more than $75 \%$ of her body.

Notes: The event was resolved by treatment with steroids followed by a slow steady taper over one month.

Photo courtesy of Jeffrey Weber MD, PhD. for life-threatening, immune-mediated dermatitis, such as generalized exfoliative, full thickness dermal ulceration, ulcerative or bullous dermatitis, skin necrosis, StevensJohnson Syndrome, or toxic epidermal necrolysis, and systemic corticosteroids at a dose of $1-2 \mathrm{mg} / \mathrm{kg} /$ day of prednisone or equivalent should be administered. When dermatitis is controlled, a corticosteroid taper should be initiated and continued over at least one month. ${ }^{19}$

\section{Gastrointestinal immune-related AEs}

\section{Case study 2: immune-related enterocolitis}

A 38-year-old woman developed a new mole on her right hand 4 years prior to a visit with a surgical oncologist in January 2009. Although the mole was changing in shape and it had developed a scab, the patient delayed medical consultation. A biopsy revealed an ulcerated, Clark's level IV lesion with a Breslow thickness of $2.5 \mathrm{~mm}$. Wide excision and sentinel lymph node biopsy of the right axilla was positive for melanoma in 2/2 nodes, American Joint Committee on Cancer (AJCC) Stage IIIb-pT3, N2a, MO. A lymph node dissection revealed 14 negative nodes. The patient was enrolled in a randomized Phase III clinical study of ipilimumab versus placebo in the adjuvant setting. One month following the second ipilimumab dose, the patient reported dysuria and a small amount of bright red blood per rectum on paper, without diarrhea. The patient was referred to a gastroenterologist and she declined hospital admission at that time. One month later, she was admitted to a local emergency room with intractable nausea, vomiting, and diarrhea. Colonoscopy revealed paninflammatory colitis with grade 3 diarrhea. She was started on methylprednisolone sodium succinate via intravenous (Solu-Medrol ${ }^{\circledR}$, Pfizer, New York, $\mathrm{NY}$ ) at a dose of $1 \mathrm{mg} / \mathrm{kg} / \mathrm{day}$. Following methylprednisolone sodium succinate administration, the patient's stools began decreasing and she was discharged on a tapering dose of oral prednisone $60 \mathrm{mg}$ daily for one week, with a $20 \mathrm{mg}$ decrease 
scheduled one week later. She developed increased diarrhea with incontinence, nausea, and vomiting. The patient was seen in a clinic and presented with an astonishing $20 \mathrm{lb}$ weight loss since her last visit. The patient was again admitted to the hospital and treated with intravenous methylprednisolone sodium succinate at a dose of $1 \mathrm{mg} / \mathrm{kg} /$ day. Since the diarrhea was persistent, the patient was given two doses of infliximab (Remicade $^{\circledR}$, Centocor Ortho Biotech Inc, Malvern, PA). She was discharged on oral steroids at a dose of $100 \mathrm{mg}$ /day with a very slow 7-week taper schedule and close monitoring. The patient failed to attend for follow-up. One month following her discharge, she was admitted with a gastrointestinal bleed and colonoscopy revealed significant proctocolitis. An appointment was made with the clinic; however, the patient failed to attend her scheduled visit. This patient presented with a severe and protracted case of immune-related colitis, but failed to comply with her treatment plan. Patient education and assessment of willingness to adhere to recommendations is very important in the use of immunotherapy.

\section{Management}

The most common immune-related AEs that affect the gastrointestinal tract manifest as diarrhea or colitis. Symptoms of gastrointestinal immune-related AEs include any changes in normal bowel habits or changes from baseline (eg, last week, last visit), diarrhea, abdominal pain, blood or mucus in the stool with or without fever, peritoneal signs consistent with bowel perforation, and ileus. Grade 2 gastrointestinal immune-related AEs are described as up to six stools above baseline, accompanied by abdominal pain and mucus or blood in stool. Severe or life-threatening gastrointestinal immune-related AEs (grades 3-4) are characterized as diarrhea of at least seven stools above baseline, along with fever, ileus, and/or peritoneal signs. ${ }^{19}$ The incidence of lifethreatening perforation is rare; signs include diffuse colitis with crypt abscess formation as visualized by colonoscopic biopsy, following onset of severe diarrhea. In symptomatic patients, rule out infectious etiologies and consider endoscopic evaluation for persistent or severe symptoms.

To manage immune-related AEs of the gastrointestinal tract, patients should be advised to report changes in bowel movements immediately. Patients need to be monitored for gastrointestinal signs and symptoms as outlined above at each follow-up visit. For grades 3-5 gastrointestinal immune-related AEs, patients should be treated with highdose corticosteroids, as above, with a taper over at least one month once symptoms are controlled. Infliximab may be administered with moderate, severe, or life-threatening gastrointestinal immune-related AEs if they do not respond to corticosteroid treatment. Ipilimumab treatment should be withheld in the event of moderate gastrointestinal immunerelated AEs until improvement to mild severity or complete resolution. Ipilimumab should be permanently discontinued if the patient experiences severe or life-threatening enterocolitis. $^{21}$

\section{Hepatic immune-related AEs Case study 3: immune-related hepatitis}

A 54-year-old woman with a changing mole in June of 2009 had a biopsy revealing a Breslow thickness $0.65 \mathrm{~mm}$, Clark's level III, nonulcerated, malignant melanoma. A right submandibular mass and a scalp nodule were also positive for melanoma. A positron emission tomography/computed tomography scan revealed a right lung nodule and a liver nodule, being AJCC stage IV melanoma. She was offered a randomized, clinical trial and received ipilimumab at the experimental higher dose of $10 \mathrm{mg} / \mathrm{kg}$.

The patient's laboratory tests were followed closely throughout ipilimumab treatment, which were normal until her alanine and aspartate aminotranferase levels spiked to more than five times the upper limit of normal, suggestive of immune-mediated hepatitis. She was already on prednisone due to a previous immune-related adverse event, but in the course of tapering off, the dose was increased again at this time. Taper was reinitiated once the aspartate and alanine aminotranferase levels had normalized. She experienced further flares in liver function tests, requiring re-escalation of her prednisone dose. Liver function tests were checked every 3 days. Eventually, her alanine and aspartate aminotranferase levels were within normal limits, and she was successfully tapered off steroids after 5 months.

Although immune-related hepatitis developed and was a persistent problem requiring careful monitoring, with dose adjustments of prednisone as needed based on her laboratory test results, the patient was eventually able to achieve resolution of her immune-related AEs. Patient compliance in this case was also critical to resolution of symptoms for a positive outcome. This patient reported feeling well during this entire time frame and was able to work and maintain all her activities of daily living.

\section{Management}

Hepatic immune-related AEs were relatively rare in the MDX010-020 study, in which patients were given ipilimumab as monotherapy ( $3 \mathrm{mg} / \mathrm{kg}$, Table 1A). However, in a recent study in which patients were treated with a combination 
of dacarbazine and ipilimumab at an experimental dose of $10 \mathrm{mg} / \mathrm{kg}$, hepatic events occurred at a much higher rate than previously observed. ${ }^{16,18}$

This suggests that the types of immune-related AEs that patients experience are predictable, but the rates of any specific event may change based on the exact regimen and other therapies that are used in combination with ipilimumab. Further research is needed to elucidate this issue.

Hepatitis following treatment with ipilimumab presents asymptomatically in patients as a rise in hepatic alanine and aspartate aminotranferase, as in the above case, with a lesser rise in bilirubin. Liver function tests, including hepatic transaminase and bilirubin levels, require monitoring and assessment for signs and symptoms of hepatitis before each dose of ipilimumab. Unlike classic hepatitis, whereby patients present with jaundice or fever, these patients are asymptomatic and thus it is critical to monitor liver function tests prior to each dose. Dosing should be withheld in patients with grade 2 hepatic toxicity, defined as moderate alanine or aspartate aminotranferase elevations of $>2.5-5.0$ times the upper limit of normal, or moderate total bilirubin elevation of $>1.5-3.0$ times the upper limit of normal. Ipilimumab should be permanently discontinued in patients who experience grade 3-4 hepatotoxicity, defined as aspartate or alanine aminotranferase elevations of more than five times the upper limit of normal or total bilirubin elevations of more than three times the upper limit of normal, and systemic corticosteroids at a dose of $1-2 \mathrm{mg} / \mathrm{kg} /$ day of prednisone or equivalent should be administered. When liver function tests show sustained improvement or a return to baseline, a corticosteroid taper should be initiated and continued over one month. Mycophenolate treatment can be administered in patients who have persistent severe hepatitis despite highdose corticosteroid treatment. ${ }^{19}$

\section{Endocrine immune-related AEs Case study 4: immune-related endocrinopathy (hypophysitis)}

A 50-year-old man with a history of metastatic melanoma with resected brain metastasis post whole-brain radiotherapy presented with a lung metastasis and a pancreatic mass after failing temozolomide (Temodar ${ }^{\circledR}$, Merck and Co, Inc, Whitehouse Station, NJ). He began an ipilimumab trial, receiving an experimental higher dose of $10 \mathrm{mg} / \mathrm{kg}$ as salvage therapy. He received the drug every 3 weeks for four doses, followed by maintenance every 3 months. After 3 doses, the patient was seen in the emergency room with symptoms of severe fatigue, cognitive dysfunction, and headache.
His thyroid-stimulating hormone level was normal, but his T3 and free T4 levels were extremely low, and he was started on thyroid replacement therapy. After his fourth dose of ipilimumab, he notified the clinic of fatigue and myalgias. Corticotropin and cortisol levels were found to be normal, but testosterone was abnormally low at 215 (normal range 286-1510). He was treated with testosterone gel (Androgel ${ }^{\circledR}$, Abbott Laboratories, North Chicago, IL). Two weeks later, the patient's cortisol level began to fall and he began to have difficulty with word finding. Prednisone at $1 \mathrm{mg} / \mathrm{kg} /$ day was started, and subsequent doses of ipilimumab were withheld while the patient was monitored for endocrinopathies. Week 12 scans revealed regression of pulmonary nodules and complete resolution of the pancreatic lesion. Steroids and testosterone gel were tapered down slowly, as tolerated by the patient. However, the patient did describe worsening of fatigue and difficulty with word finding. He required re-escalation of prednisone. For approximately a year and a half, he continued monitoring while being treated with prednisone, testosterone gel, and thyroid medication. The patient was able to eventually restart ipilimumab treatment and continues in a clinical trial focused on maintenance therapy with ipilimumab. His latest scans confirm stable response.

\section{Management}

Endocrinopathies may resolve or may be a permanent adverse effect requiring long-term steroid therapy, as well as thyroid and testosterone replacement. Endocrine immune-related AEs occurred in 4\%-8\% of patients treated with ipilimumab in the MDX010-20 trial (Table 1A). ${ }^{16}$ Endocrinopathies experienced by patients taking ipilimumab are often characterized by presentation of fatigue and/or headache, change in mental status, or symptoms of hypothyroidism. Further, patients may present with nonspecific symptoms resembling other causes, such as brain metastases or progression of underlying disease. Thyroid function tests and clinical biochemistry should be monitored at the start of treatment and before each dose of ipilimumab, as well as subsequent to therapy, if symptomatic. Severe to life-threatening endocrinopathies (grades 3-4) manifest as hypopituitarism, adrenal insufficiency (including adrenal crisis), and hyperthyroidism or hypothyroidism. ${ }^{19}$ To manage endocrine immune-related AEs, patients should be advised to report symptoms immediately. An endocrine panel should be drawn, with analysis of corticotropin, cortisol, T3, T4, thyroid-stimulating hormone, and testosterone levels for men, and the same panel with follicle-stimulating hormone and prolactin for women. A magnetic resonance imaging scan of the brain with pituitary cuts should be performed. 
Ipilimumab should be withheld in symptomatic patients, and systemic corticosteroids at a dose of $1-2 \mathrm{mg} / \mathrm{kg} /$ day of prednisone or equivalent should be administered and hormone replacement therapy initiated. ${ }^{19}$

\section{Other immune-related AEs Neurologic immune-related AEs}

Serious and fatal immune-mediated neurologic adverse reactions associated with ipilimumab use include sensory and motor neuropathy, Guillain-Barré syndrome, and myasthenia gravis. Severe to fatal immune-related neuropathies were rare, and experienced by fewer than $2 \%$ of patients receiving ipilimumab in the MDX010-20 study. ${ }^{19}$ To facilitate management of neurologic immune-related AEs experienced by patients receiving ipilimumab, patients should be advised to report signs or symptoms such as muscle weakness or sensory alterations immediately. Patients need to be monitored for symptoms of motor and sensory neuropathy, unilateral or bilateral weakness, sensory alterations, and paresthesia. ${ }^{19}$ Ipilimumab should be withheld in patients who experience moderate neuropathy (not interfering with daily activities), and should be permanently discontinued in patients with severe neuropathy, such as Guillain-Barré-like syndrome. Medical intervention needs to be instituted for management of severe neuropathy, including prednisone $1-2 \mathrm{mg} / \mathrm{kg} /$ day. ${ }^{19}$

\section{Ocular immune-related AEs}

Although very rare, some patients have experienced ocular events. Corticosteroid eye drops are recommended for patients who develop uveitis, iritis, or episcleritis. It is recommended that ipilimumab be permanently discontinued in any patient experiencing ocular events that are unresponsive to local topical therapy. ${ }^{19}$

\section{General information}

For patients experiencing any of the immune-related AEs described above while taking ipilimumab, treatment should be discontinued if the prescriber is unable to reduce the corticosteroid dose to $7.5 \mathrm{mg}$ prednisone or equivalent per day, or if the patient is unable to complete the full treatment course within 16 weeks from administration of the first dose of the drug. ${ }^{19}$

\section{Nursing checklist for immune- related AEs}

A sample nurse's questionnaire is presented (Figure 2) with pertinent questions on immune-related AE symptoms; this questionnaire can guide the topics that should be covered with a patient prior to starting treatment and reinforced throughout the course of the therapy. Additional information and materials can be found through the Risk Evaluation and Mitigation Strategy program (http://www.yervoy.com/hcp/ pdf/rems-nursing-checklist.pdf). Patients and their caregivers should be encouraged to report symptoms early so that the patient can continue to reap the benefits of ipilimumab.

\section{Patterns of response with ipilimumab}

In addition to the safety events, which may be tied to the unique mechanism of action of ipilimumab, unique clinical responses might also be reflective of the immune-related mechanism of action. (Table 2) Response patterns with ipilimumab include two standard responses that are commonly observed with other cytotoxic therapies, the first being an immediate decline in overall tumor burden with no new lesions and the second being stable disease. Stable disease in some patients on ipilimumab can be followed by a slow and steady decline in tumor burden. ${ }^{17,19,22,23}$

However, throughout clinical development of ipilimumab, two additional, novel response patterns have been observed in patients, while still being shown to be associated with improved survival in patients (Table 2). The first is a reduction in overall tumor burden in the presence of new lesions and the second is an initial increase followed by a steady decrease in tumor volume. ${ }^{22}$ Understandably, both can be concerning for patients and their caregivers, because an initial increase or presence of new lesions might be a sign that the treatment is not working. It is important that patients understand that responses may take time to appear (ie, the immune system needs time to work) and that what appears to be progressive disease initially may not be actual progressive disease. It is

Table 2 Patterns of response with ipilimumab 17,19,22,23

- Immediate response in baseline lesions, without the presence of new lesions

- Durable stable disease (SD), which may be followed by a slow, steady decline in total tumor burden

- Response after an increase in total tumor burden

- Response in presence of new lesions (which may have been present at baseline but were radiographically undetectable)

Notes: All patterns of response have been associated with response in patients and to improved survival. 


\begin{tabular}{|c|c|}
\hline $\begin{array}{l}\text { Ask patients about their signs and } \\
\text { symptoms }\end{array}$ & $\begin{array}{l}\text { Yes answers may indicate the } \\
\text { patient is experiencing an irAE }\end{array}$ \\
\hline $\begin{array}{l}\text { Are you experiencing any diarrhea, } \\
\text { increased bowel movements, watery } \\
\text { stools, or any cramping or pain in your } \\
\text { belly? }\end{array}$ & Gastrointestinal irAEs \\
\hline $\begin{array}{l}\text { Have you been having a hard time } \\
\text { sleeping or feeling sleepier than usual? } \\
\text { Are you experiencing headaches, light- } \\
\text { headedness, or changes in mood? }\end{array}$ & Endocrine irAEs \\
\hline $\begin{array}{l}\text { Does your skin feel itchy anywhere, or } \\
\text { have you noticed any new rashes, or } \\
\text { any changes in pigmentation? }\end{array}$ & Dermatologic irAEs \\
\hline $\begin{array}{l}\text { Have you noticed any weakness or } \\
\text { trouble gripping or dropping things? Do } \\
\text { you have tingling in your fingers or } \\
\text { toes? }\end{array}$ & Neurologic irAEs \\
\hline $\begin{array}{l}\text { Have you noticed any changes in } \\
\text { vision or problems with your eyes? }\end{array}$ & Ocular irAEs \\
\hline
\end{tabular}

Figure 2 Questionnaire for nurses to guide discussions with patients on ipilimumab therapy.

necessary to re-evaluate patients with progressive disease or a mixed response 4 weeks after the first evaluation in order to determine the future course of therapy in the patient. ${ }^{22}$

\section{Discussion}

Ipilimumab is a novel immunotherapeutic agent approved by the FDA for unresectable and metastatic melanoma. Due to its characteristic and distinctive mechanism of action, ipilimumab elicits a number of specific immune-related AEs. Time to onset and resolution of ipilimumab-associated immune-related AEs follow a predictable temporal pattern but can vary from patient to patient. Nevertheless, guidelines for management of ipilimumab-associated immune-related AEs and a nurse's checklist for signs and symptoms of immune-related AEs are now provided by the manufacturer and can greatly assist health care providers in identifying and treating such events. Expedient and careful monitoring of immune-related AEs and nurses' recognition of possible AEs that may occur during treatment with ipilimumab may allow patients to continue to benefit from therapy and optimize outcomes.

\section{Acknowledgments}

The authors would like to thank Jeffrey Weber MD, $\mathrm{PhD}$ for providing the patient photograph demonstrating skin toxicity.

\section{Disclosure}

The authors also wish to acknowledge StemScientific, funded by Bristol-Myers Squibb, for providing editorial 
support for the print manuscript and video abstract.. Neither Bristol-Myers Squibb nor StemScientific influenced the content of print manuscript or video, nor did the authors receive financial compensation for authorship.

\section{References}

1. Jemal A, Siegel R, Xu J, Ward E. Cancer statistics, 2010. CA Cancer J Clin. 2010;60:277-300.

2. Siegel R, Ward E, Brawley O, Jemal A. Cancer statistics, 2011: the impact of eliminating socioeconomic and racial disparities on premature cancer deaths. CA Cancer J Clin. 2011;61:212-236.

3. Howlader N, Noone AM, Krapcho M, et al, editors. SEER Cancer Statistics Review, 1975-2008, National Cancer Institute. Bethesda, MD. Available from: http://seer.cancer.gov/csr/1975_2008/. Accessed September 20, 2011

4. Balch CM, Gershenwald JE, Soong SJ, et al. Final version of 2009 AJCC melanoma staging and classification. J Clin Oncol. 2009;27: 6199-6206.

5. National Comprehensive Cancer Network. Melanoma. 2012. Available from: http://www.nccn.org/index.asp. Accessed October 28, 2011

6. Barth A, Wanek LA, Morton DL. Prognostic factors in 1,521 melanoma patients with distant metastases. J Am Coll Surg. 1995;181: 193-201.

7. Hodi FS, Oble DA, Drappatz J, et al. CTLA-4 blockade with ipilimumab induces significant clinical benefit in a female with melanoma metastases to the CNS. Nat Clin Pract Oncol. 2008;5:557-561.

8. Margolin KA, Di Giacomo AM, Maio M. Brain metastasis in melanoma: clinical activity of CTLA-4 antibody therapy. Semin Oncol. 2010;37: $468-472$.

9. Radbill AE, Fiveash JF, Falkenberg ET, et al. Initial treatment of melanoma brain metastases using gamma knife radiosurgery: an evaluation of efficacy and toxicity. Cancer. 2004;101:825-833.

10. Staudt M, Lasithiotakis K, Leiter U, et al. Determinants of survival in patients with brain metastases from cutaneous melanoma. Br J Cancer. 2010;10:1213-1218
11. Dummer R, Maio M, Hamid O, et al. Abstract P-0004 presented at the Perspectives in Melanoma XIV meeting, Amsterdam, The Netherlands; September 17-18, 2010.

12. Ives NJ, Stowe RL, Lorigan P, Wheatley K. Chemotherapy compared with biochemotherapy for the treatment of metastatic melanoma: a metaanalysis of 18 trials involving 2,621 patients. J Clin Oncol. 2007;25: $5426-5434$.

13. Agarwala SS. Current systemic therapy for metastatic melanoma. Expert Rev Anticancer Ther. 2009;9:587-595.

14. Chapman PB, Hauschild A, Robert C, et al. Improved survival with vemurafenib in melanoma with BRAF V600E mutation. $N$ Engl J Med. 2011;364:2507-2516.

15. Zelboraf ${ }^{\circledR}$ (vemurafenib) package insert. South San Francisco, CA: Genentech USA Inc,; 2011.

16. Hodi FS, O'Day SJ, McDermott DF, et al. Improved survival with ipilimumab in patients with metastatic melanoma. NEngl J Med. 2010; 363:711-723.

17. Hoos A, Ibrahim R, Korman A, et al. Development of ipilimumab: contribution to a new paradigm for cancer immunotherapy. Semin Oncol. 2010;37(5):533-546.

18. Robert C, Thomas L, Bondarenko I, et al. Ipilimumab plus dacarbazine for previously untreated metastatic melanoma. N Engl J Med. 2011; $364: 2517-2526$

19. Yervoy ${ }^{\mathrm{TM}}$ (ipilimumab) package insert. Princeton, NJ: Bristol-Myers Squibb Company; 2011.

20. Ibrahim R, Berman D, de Pril V, et al. Ipilimumab safety profile: summary of findings from completed trials in advanced melanoma. J Clin Oncol. 2011;29 Suppl:Abstract 8583.

21. Bristol-Myers Squibb. Ipilimumab US prescribing information: risk evaluation and mitigation strategy (REMS). Available from: http:// www.yervoy.com/hcp/rems.aspx. Accessed September 12, 2011.

22. Wolchok JD, Hoos A, O'Day S, et al. Guidelines for the evaluation of immune therapy activity in solid tumors: immune-related response criteria. Clin Cancer Res. 2009;15:7412-7420.

23. Hoos A, Eggermont AM, Janetzki S, et al. Improved endpoints for cancer immunotherapy trials. J Natl Cancer Inst. 2010;102:1388-1397.
Cancer Management and Research

\section{Publish your work in this journal}

Cancer Management and Research is an international, peer-reviewed open access journal focusing on cancer research and the optimal use of preventative and integrated treatment interventions to achieve improved outcomes, enhanced survival and quality of life for the cancer patient The journal welcomes original research, clinical \& epidemiological

\section{Dovepress}

studies, reviews \& evaluations, guidelines, expert opinion \& commentary, case reports \& extended reports. The manuscript management system is completely online and includes a very quick and fair peerreview system, which is all easy to use. Visit http://www.dovepress.com/ testimonials.php to read real quotes from published authors. 